\title{
VIENTOS DE CAMBIOS. EL CASO DE LA MUTUAL FEDERADA SALUD
}

\author{
Kobila, María Teresa \\ Parolin, Marisa Andrea \\ Chiaramoni, Natalia Soledad \\ D’Avanzo, María Laura \\ Gagliardini, Graciela Ana
}

\section{RESUMEN:}

Las organizaciones post-Covid 2019 plantean problemáticas y desafíos abriendo un abanico de oportunidades para el apasionante mundo de la investigación. La Asociación Mutual Federada Salud se seleccionó como "caso de estudio único". Se empleó metodología cualitativa y la entrevista virtual fueron el principal instrumento de relevamiento, proyectándose el abordaje de los siguientes objetivos: describir los cambios que se han implementado a partir del contexto de pandemia, reseñar las políticas y/o estrategias aplicadas, básicamente sobre el talento humano, inferir el estilo de gestión prevalente en la mutual y reflexionar sobre el proceso de cambio operado en la organización. Se reflexiona que los cambios fueron resultantes de decisiones políticas, sin las cuales su implementación hubiera sido imposible. Se procuró acrecentar las fortalezas y estrechar las debilidades para hacer operativos los cambios pretendidos que se dispuso mediante el empleo de tecnologías propias y el reforzamiento sistemático en la gestión del talento humano. El proceso de cambio no fue impulsado por la pandemia si bien se aceleró a consecuencia de la misma. La redefinición de valores fue ineludible para orientar el rumbo organizacional. La claridad en los compromisos de trabajo, la orientación a resultados específicos, la adaptación a los ambientes personales e institucionales, demuestra que el cambio es posible. Se infiere que el Gerente General-líder posee un "estilo mixto" que combina capacidades de gerenciar y liderar. Este proceso se interpreta como un auténtico cambio cultural, que se inició con un diagnóstico de la situación existente y llevó a redefinir los valores compartidos y consensuados por todos de los miembros en pos de la construcción conjunta de una floreciente y apropiada cultura organizacional.

PALABRAS CLAVE: Gestión del cambio. Gestión del Talento Humano. Liderazgo. Cultura organizacional

\section{ABSTRACT:}

Post-Covid 2019 organizations has problems and challenges, opening a range of opportunities for the exciting world of research. Mutual Federada Salud Association was selected as a "unique case study". Qualitative methodology was used and the virtual 
interview was the main survey instrument, projecting the approach to the following objectives: describe the changes that have been implemented from the pandemic context, review the applied policies and / or strategies, basically on talent human, infer the prevalent management style in the mutual and reflect on the process of change operated in the organization. It reflects that the changes were the result of political decisions, without which their implementation would have been impossible. It was treated made to increase the strengths and narrow the weaknesses to make operational the intended changes that were arranged through the use of proprietary technologies and the systematic reinforcement in the management of human talent. The process of change was not driven by the pandemic, although it accelerated as a result of it. The redefinition of values was unavoidable to guide the organizational course. Clarity in work commitments, orientation to specific results, adaptation to personal and institutional environments, shows that change is possible. It is inferred that the General Manager-leader has a "mixed style" that combines management and leadership skills. This process is interpreted as an authentic cultural change, which began with a diagnosis of the existing situation and led to a redefinition of shared values and agreed by all of the members in post of the joint construction of a flourishing and appropriate organizational culture.

KEY WORDS: Change management. Management of human talent. Leadership. Organizational culture.

\section{INTRODUCCIÓN}

A nivel social, político y económico el Covid-19 fue el protagonista del año 2020, con sus consecuencias de confinamientos, crisis y diversas secuelas a nivel mundial, regional y local. La pandemia aún no se ha controlado, pero sin lugar a dudas dejarán marcas por un largo período de tiempo. Para muchos, un cambio de paradigma.

Las organizaciones argentinas no están al margen de la realidad mundial, aunque a pesar de los cambios externos e internos, no todas corrieron la misma suerte. La resistencia y supervivencia de algunas de ellas se sospecha derivadas de la prevalencia de ciertos estilos de conducción y pautas culturales. Estas interpretaciones fueron vertidas en un trabajo de investigación realizado en el año $2020^{1}$.

Por entonces, conscientes de la creciente complejidad en sus múltiples dimensiones, observamos, interpretamos y describimos la sucesión acelerada de cambios significativos y diversos que dieron cuenta de las reacciones y posibles respuestas frente a lo inesperado.

1"iEureka! Se aceleran los cambios organizacionales con la pandemia” presentado en las V Jornadas de Investigar para Investigar, Facultad de Ciencias Económicas y Estadística de la U.N.R., octubre de 2020. 
Muchos supusieron que los cambios -en las tecnologías, las estructuras y conductas humanas- llegaron para quedarse, otros imaginaron que sería algo temporal y también están quiénes juzgaron que prevalecerían las modalidades laborales híbridas.

Sin dudas, las organizaciones post-Covid 2019 plantean problemáticas y desafíos abriendo un abanico de oportunidades para el apasionante mundo de la investigación. De cara a este estado de situación, atendiendo las opiniones del mundo empresarial y con la ambición de ampliar los conocimientos en el campo de la Administración se gestó y construyó este trabajo.

\section{SITUACIÓN PROBLEMÁTICA}

Indiscutiblemente en el ámbito organizacional, dónde los líderes y sus colaboradores son piezas claves, se vivenciaron cambios importantes, interpelando a sus propios protagonistas. Estos aconteceres arrojaron disparadores que exhortan a averiguar si ¿el pensar de los directivos es congruente con el comportamiento esperado de sus colaboradores? Los cambios implementados ¿siguen interpelando mayores desafíos a futuro? En relación, concierne indagar ¿Cuál es la proyección que tiene la organización? ¿Cuáles son las medidas consideradas urgentes y/o necesarias para la continuidad organizacional? ¿Cómo es considerada la dimensión de capital humano para el/los directivos?

Con el convencimiento de que los directivos que aspiran al progreso organizacional orientan su mirada hacia el personal, atañe comprender ¿cómo es gestionado el talento humano? ¿Existen políticas, estrategias o procedimientos con relación al desarrollo del talento humano? Indagaciones que orientan el trabajo de investigación.

Se estudia una organización mutual -comentado en el apartado de metodología- cuyas dimensiones culturales fueron abordadas por nuestro equipo en el año 2014 ${ }^{1}$. La decisión de ampliar esta indagatoria obedece al interés que el caso de estudio inspira. En el marco de nuevo contexto se plantearon nuevos interrogantes y los siguientes objetivos de investigación.

\section{OBJETIVOS}

a) Describir los cambios que se han implementado a partir del contexto de pandemia.

c) Reseñar las políticas y/o estrategias aplicadas, básicamente sobre talento humano.

b) Inferir el estilo de gestión prevalente en la mutual.

\footnotetext{
${ }^{1}$ Decimonovenas Jornadas de Investigaciones en la Facultad de Ciencias Económicas y Estadística, realizadas los días 27 y 28 de Noviembre de 2014. Disponible en:

https://rephip.unr.edu.ar/handle/2133/7414/discover
} 
b) Reflexionar sobre el proceso de cambio operado en la organización.

\section{METODOLOGÍA}

Esta ponencia está enmarcada en el proyecto "Gestión de la complejidad cultural de las organizaciones" ${ }^{1}$. En esta línea de investigación descuella la metodología cualitativa ${ }^{2}$ a través de la cual se aspira descubrir los supuestos del estudio para la reconstrucción de los datos. La Asociación Mutual Federada Salud se seleccionó como "caso de estudio único"3a consecuencia de las preguntas orientadoras y los objetivos indicados precedentemente que fueron parte del diseño de investigación.

Consiguientemente la entrevista fue el instrumento de relevamiento básico seguido por la revisión documental con un diseño bibliográfico basado en la revisión sistemática de la base de datos que almacenaba el equipo de investigación. Las entrevistas se efectuaron bajo la modalidad virtual a través del dispositivo google meet. Después del relevamiento se procedió a la transcripción de las mismas para su posterior análisis. Se dispusieron preguntas abiertas en un guion que destacó consultas en torno a la formulación de las palabras clave, centro de interés de este trabajo.

El relevamiento se efectuó durante los meses de julio y agosto de 2021, las entrevistas en profundidad se mantuvieron con el gerente general de la mutual quién proporcionó valiosa información, sin ocultación de información ni límites de tiempos. Es de destacar que en redacción y el sentido único de las expresiones lingüísticas se respetaron los dichos del entrevistado, en ese sentido los resultados se construyen en conexión con los comentarios vertidos y la interpretación efectuada.

Finalmente, conviene recordar que se trata de un estudio de "caso único" donde no es factible la extrapolación de resultados a otros casos $u$ organizaciones.

\section{MARCO TEÓRICO}

Como se esbozó en el apartado introductorio, el comienzo de la pandemia COVID-19 ha expuesto al mundo a un complicado desafío. Las estrategias de contención anunciados por los gobiernos hicieron cambiar a la gente a modelos de trabajo remoto del día a la noche. Aun antes del estallido de la pandemia, la adopción y la evolución de la tecnología, los modelos de trabajo y el comportamiento del consumidor estaban cambiando. Sin embargo,

\footnotetext{
${ }_{1}^{1}$ Proyecto radicado en la Facultad de Ciencias Económicas y Estadística de la U.N.R., período20202021.

${ }^{2}$ Hernández Sampieri y otros. (2010) Metodología de la Investigación. Buenos Aires.

${ }^{3}$ Yin, R. K. (2004) Investigación sobre estudio de casos. Sage Publications: México: Sage Publications.
} 
el repentino comienzo del COVID ha intensificado el uso de herramientas de tecnología de la nueva era ${ }^{1}$.

Para gestionar los cambios, la cultura organizacional y el talento humano se requieren el impulso de líderes. El líder efectivo tiende a ubicarse en algún lugar de la intersección de estos temas. Peter Drucker ${ }^{2}$ sostiene que lo que valida la existencia de un líder es que tenga seguidores y $\mathrm{Kotter}^{3}$ agrega "El liderazgo complementa a la gestión, no la sustituye". Como expresa Sarchman ${ }^{4}$ para fortalecer las capacidades sociales y emocionales y dirigir el cambio el talento de los líderes es fundamental.

Atesorar el talento humano se ha convertido en uno de los principales retos de los directivos en un cada vez más turbulento entorno de negocios. El talento de los gerentes es necesario para rediseñar estrategias que se enfoquen en las capacidades fundamentales que puedan traer resultados mejorados. Si los equipos de talento fracasan para nutrir el talento existente de sus empleados, potencialmente limitan el crecimiento y la sustentabilidad de la organización en el futuro.

Un reportaje realizado a Orlando Mejía ${ }^{5}$, socio de Capital Humano de Deloitte, expresa: "La pandemia y la realidad del futuro del trabajo que están ocurriendo ahora van a llevar a que Recursos Humanos tome su lugar en la mesa, igual o más importante que el de Finanzas o el de Marketing". Para florecer durante y después de la crisis, los empleados deben estar equipados para asegurar adaptabilidad, flexibilidad y creatividad.

La reinvención del Área de Recursos Humanos, es "la clave" para el mundo laboral postpandemia. En los ámbitos organizacionales la emergencia sanitaria constriñe a las áreas de gestión de talento a replantear sus funciones dentro de las empresas para que asuman el liderazgo y orienten a sus colaboradores para trabajar bajo la "nueva normalidad". Como sostiene Sarchman ${ }^{6}$ el talento de los gerentes se necesita para rediseñar estrategias que se enfoquen en las capacidades fundamentales que puedan traer resultados mejorados. Si los equipos de talento fracasan, potencialmente limitan el crecimiento y la sustentabilidad de la organización en el futuro.

La gestión del talento humano, engloba el conjunto de políticas y prácticas necesarias para dirigir el desarrollo de los cargos gerenciales, siendo esencial para las organizaciones que

\footnotetext{
1 Sarchman, José M. (2021) "Habilidades que requieren las empresas en tiempos de pandemias", recuperado el 30 de julio de 2021 de: :

http://www.biblioeco.unsa.edu.ar/pmb/opac css/index.php?|v|=notice display\&id=52538

${ }^{2}$ Drucker, P. (1999) Los desafíos de la administración en el siglo XXI. Buenos Aires: Sudamericana

${ }^{3}$ Kotter, J. P. (2002) "Lo que de verdad hacen los líderes" en: Harvard Business Review Liderazgo, Bilbao, Ed. Deusto, pp. 41.

${ }^{4}$ Ibídem.

${ }^{5}$ Redacción Factor Capital Humanos (2020, 20 julio) "Reinvención de Recursos Humanos, clave para el mundo laboral post pandemia". Recuperado el 13 de agosto de 2021, de https://factorcapitalhumano.com/mundo-del-trabajo/reinvencion-de-recursos-humanos-clave-para-elmundo-laboral-post-pandemia/2020/07/

${ }^{6}$ Op. Cit. 7
} 
consideran al talento como un activo estratégico". Para los intelectuales, la inversión y desarrollo del capital intelectual es un axioma básico, para los gestores el talento se ha convertido en una cuestión organizacional que involucra a toda la organización, y en especial a la alta dirección ${ }^{2}$

Una vez definida la estrategia por la alta dirección, ésta debe llegar de manera precisa a cada uno de los integrantes de la organización. Además, cuando cada uno de los colaboradores conoce como impacta positivamente en los resultados, esto contribuirá a la consolidación de la cultura organizacional forjando condiciones atractivas para el desarrollo del talento humano en el largo plazo.

Cejas $(2008)^{3}$ señala que este enfoque constituye un elemento clave del éxito competitivo que permite el mejoramiento del desempeño de las personas a través del desarrollo de las competencias. Cabe destacar que, las competencias deben ir acompañadas del sentido de compromiso y la acción, tal como lo plantea Alles $(2017)^{4}$, si un profesional tiene el sentido del compromiso y actúa; pero no dispone de las capacidades, evidentemente no alcanzará buenos resultados. Por el contrario, si posee las capacidades y actúa, sin comprometerse con el proyecto, puede lograr lo fijado; sin embargo, la falta de motivación le impedirán innovar.

También Peiró y Soler $(2020)^{5}$ señalan un conjunto de habilidades que han de estar presentes en el talento humano, tales como las destrezas digitales, cognitivas, emocionales, manejo de las redes, resolución de conflictos, adaptación a los cambios, entre otros. Lo cual contribuiría tanto a la empleabilidad, como al alto rendimiento en los procesos y la capacidad de responder a las realidades actuales. En tal sentido, las organizaciones han de contemplar los nuevos requerimientos del talento humano en el mundo laboral. Por ello, pareciera inexcusable realizar procesos formativos que estén soportados en herramientas tecnológicas, plataformas en líneas, redes sociales, WhatsApp, correos electrónicos, canales YouTube, entre otros.

\footnotetext{
${ }^{1}$ Chiavenato, I. (2007). "Administración de Recursos Humanos: El capital humano en las organizaciones" Buenos Aires: Mc Graw Hill

${ }^{2}$ Hatum, A. (2011). "El Futuro del Talento. Gestión del talento para sobrevivir la crisis". Buenos Aires: Temas

${ }^{3}$ Cejas, M. (2008). La Formación profesional basada en competencias. Carabobo, Venezuela: Editorial Consejo de Desarrollo Científico Humanístico de la Facultad de Ciencias Económicas y Sociales. Disponible en: http://servicio.bc.uc.edu.ve/faces/revista/a12n22/13-22-7.pdf

${ }^{4}$ Alles, M. (2017). Desarrollo del talento humano basado en competencias. España: Granica.

${ }^{5}$ Peiró, J. y Soler, A. (2020). El Impulso al Teletrabajo Durante El Covid-19 y los Retos que Plantea, recuperado el 05 de agosto de 2021 de: https://www.ivie.es/wp-

content/uploads/2020/05/11.Covid19lvieExpress.El-impulso-al-teletrabajo-durante-el-COVID-19-y-losretos-que-plantea.pdf
} 
Peter Senge ${ }^{1}$ entiende que hoy se necesitan "organizaciones inteligentes", es decir que tengan perspectivas claras y posean información y conocimientos, dado que la realidad actual destruye la ilusión de que el mundo está compuesto por fuerzas separadas y desconectadas y para construir organizaciones inteligentes se debe abandonar dicha ilusión. Se requiere de organizaciones donde la gente expanda continuamente su aptitud para crear los resultados que desea, donde se cultiven nuevos y expansivos patrones de pensamiento, donde la aspiración colectiva queda en libertad, y donde la gente continuamente aprende a aprender en conjunto.

Las organizaciones que cobrarán relevancia en el futuro serán las que descubran cómo aprovechar el entusiasmo y la capacidad de aprendizaje de la gente en todos los niveles de la organización.

\section{RESULTADOS}

\section{El caso de estudio}

La Asociación Mutual Federada fue fundada en Rosario en el año 1963 y comenzó a gestionar bajo una gestión no profesionalizada. Una década más tarde la organización atravesó un momento crítico desde el punto de vista financiero, pero logró sanear el inconveniente gracias al aporte voluntario de alrededor cinco mil asociados. En la década de los ochenta se abrió a la comunidad incorporando nuevos servicios, tecnologías e incrementando notoriamente el número de asociados y después del período de convertibilidad, la mutual empezó a crecer a un ritmo constante procurando adaptarse a los cambios.

En Rosario, provincia de Santa Fe, República Argentina se halla la sede central de esta prestigiosa institución. Es apreciable la participación, a través de sus representantes políticos, de entidades de segundo grado (CEMPRA -Cámara de Empresas de Medicina Prepaga de la República Argentina) y tercer grado (UAS- Unión Argentina de Salud). Cuenta con una amplia red de delegaciones, oficinas y agencias por medio de las cuales alcanza una cobertura territorial en diferentes provincias llevando adelante una firme política expansiva.

Otra destacada fortaleza radica en el entusiasmo por querer mejorar el estado de situación, ratificado por la articulación y el trabajo conjunto entre el nivel político y de gestión partiendo de un análisis FODA que realiza la organización, acentuando la mirada hacia el interior de la misma.

En sus cincuenta y ocho años de historia logró completar asociativamente proyectos muy importantes tales como, Compañía de Seguros de Personas (Federada Seguros en

\footnotetext{
${ }^{1}$ Senge, P. (1992). La quinta disciplina. El arte y la práctica de la organización abierta al aprendizaje. España: Granica.
} 
asociación con la Mutua Divina Pastora de Valencia España), o Solidez (es una sociedad anónima que desde el año 1996 comparten con Medifé y Ase para dar cobertura a la alta complejidad en salud).

En junio de 2019 el actual gerente general ingresa a la organización, después de un período de dieciocho meses en que ese cargo estuviera vacante. La gestión de Federada era llevada adelante por el Consejo Directivo, es decir el órgano político. Hasta entonces los puestos claves habían sido ocupados por personas con trayectoria en la organización pero que nunca habían sido evaluados en relación al desempeño en esos cargos.

Por entonces, no existía la Gerencia de Desarrollo Humano a pesar que la mutual posee casi seiscientos colaboradores. Sólo existía una oficina de personal que liquidaba sueldos y registraba las novedades. Tampoco estaba la Gerencia de Prestaciones, considerada neurálgica debido a que administra el mayor presupuesto de la entidad. También, hacía casi un año que se había abandonado un outsoucing informático para desarrollar su propia área de sistemas bajo la dirección de la Gerencia de operaciones. Se carecía de auditoría interna para el control de los procesos y sólo algunos estaban sistematizados.

La nave insignia que es Federada Salud -prepaga-, es acompañada por otros verticales con desarrollos disimiles, tales como seguros, turismo, ayuda económica, farmacia y vacunatorio. La estrategia comercial era muy tímida debido a su origen como entidad sin fin de lucro. Por los comentarios vertidos se entiende que la falta de agresividad comercial lesionaba la imagen corporativa.

Entre otras debilidades se identificaron la falta de trabajos por proyecto o por objetivos. Los líderes de los mandos medios no tenían claridad respecto a los roles que debían desempeñar, observaciones que no asombran dado que habían llegado a ocupar esos cargos por antigüedad y mejoras remunerativas sin acreditación de idoneidad para el desempeño.

El diagnóstico valió para que el nivel político, junto al nuevo Gerente General trabajara en torno a las siguientes decisiones consideradas estratégicas:

- Incorporar una Gerente de Desarrollo Humano para profesionalizar el área.

- Incorporar un Gerente de Prestaciones que centralice las áreas claves de administración del presupuesto en salud tales como acuerdos (contratos con prestadores) y liquidaciones (la auditoría de la ejecución de dichos contratos).

- Emprender conjuntamente con el órgano político un proceso de reflexión estratégica que permita a la entidad redefinir su propósito, misión y visión.

- Crear el área de desarrollo organizacional para que esta administre los proyectos, brinde servicios de Project Manager para los distintos departamentos, y logre describir procesos para poder sistematizarlos. 
- Generar un comité de crisis del gasto prestacional transversal a la organización.

- $\quad$ Potenciar el área comercial redefiniendo canales de ventas y responsables.

- $\quad$ Ordenar regionalmente a la organización a través de un plan de desarrollo.

- Potenciar a la Gerencia de Auditoría Médica creando equipos interdisciplinarios como equipo de discapacidad, equipo de enfermedades crónicas, equipo de seguimiento Covid.

\section{Dinamismos durante el periodo de pandemia}

Durante todo el período de pandemia (2020-2021) se trabajó en un proceso de reflexión estratégica en pos de redefinir la misión, visión y el principal propósito de la organización. Es de destacar que la misión y visión habían sido definidas en el año 2013 y desde entonces no se habían revisado.

Según relató el entrevistado, en el proceso de reflexión estratégica participaron el Consejo Directivo conformado por diecinueve personas, trece de ellos son miembros del consejo y seis pertenecen a la junta fiscalizadora, diez gerentes y otros colaboradores de la mutual. La construcción de la misión fue un trabajo en conjunto que llevó varios meses y estuvo coordinado por una consultora externa. Se definieron cuatro valores que sirven de marco para las acciones que la organización emprende: compromiso, colaboración, cercanía y evolución.

En relación a este punto se observó que los valores citados en el año 2014, hacían referencia a: ayuda mutua, solidaridad, eficiencia, ética, transparencia, seriedad, credibilidad y el compromiso con los prestados. Si bien estos valores apoyaban la consolidación de la cultura organizacional parecían disfuncionales con la visión y propósitos de la organización que requerían profundización y esclarecimiento. Por lo tanto, se infiere que la definición actual del propósito organizacional ayuda a superar esa debilidad procedente de esa enunciación equívoca.

El propósito es "acompañar, promover y contribuir al bienestar de los asociados trascendiendo la comunidad y ser la empresa de la economía social líder en el país y la región que impulsa nuevos paradigmas de bienestar y sostenibilidad apostando en la constante búsqueda de nuevos y mejores servicios". En este sentido, el gerente aclaró:

"Somos una entidad de la economía social en este transitar a veces se confunde ser una entidad de la economía social con una cooperativa de trabajo, con algunas cuestiones de definiciones políticas del contexto entonces muchas veces hay que salir a aclarar que es una entidad de la economía social, cuáles son sus fortalezas definición así que salimos a encuestar para ver cómo nos ven los asociados y no asociados con respecto a este concepto y es muy variado hay quienes lo ven como 
una fortaleza al mutualismo, constructivismo y hay otros que lo relacionan a las cuevas financieras y lo ven como algo más turbio, no tan transparente, no tan social, la gente más joven lo ve fuera de contexto, anacrónico y la gente más del interior, comerciantes lo ven como la gran fortaleza para poder acompañar sus proyectos, es muy variado y federada abarca trece provincias, es como que hay una Federada a la carta en cada lugar y todos los lugares te van leyendo diferente" (Pablo, 17 de agosto de 2021).

Así, la encuesta permitió tener una representación más precisa de los propios miembros de la mutual acerca de la concepción de la mutual, y en base a ese relevamiento diseñar diferentes planes de acción. A posteriori, se logró una mejor articulación con los equipos interdisciplinarios, conformados por gerentes y empleados que recién comenzaban a trabajar en la organización, sirviendo de marco para conocer a las personas y potenciar el trabajo conjunto. Se interpreta que este acercamiento con todo el personal posibilitó internalizar los modos de pensar y actuar de la organización como un todo más orgánico.

Las personas que trabajan en las agencias tienen características diferentes, pero hay un aspecto que es común a todas: conocen a los asociados, sus problemáticas y la composición de su grupo familiar, de allí que esa cercanía posibilita una mayor empatía y esto también se visualiza como una fortaleza para la mutual permitiendo el mantenimiento de la cartera de asociados. También los agentes son motivados mediante una retribución por el ingreso de nuevos asociados.

Se detecta como debilidad ciertos desórdenes en la comunicación brindada por el sector comercial y el área institucional; en este aspecto se estima conveniente medir el impacto de las comunicaciones y el uso de los diferentes canales de comunicación que están en función de quién lo administra. Se decidió emprender una auditoria de comunicación interna para corregir estos desvíos. El relevamiento se propuso realizar a nivel gerencial y en todas aquellas personas quienes generan, notifican o reciben comunicación.

A partir de la pandemia se ha mantenido una cercanía con los contactos externos a través de medios virtuales, telefónicos y comunicaciones por WhatsApp. No hubo dificultades respecto del trabajo de los colaboradores que continuaron brindando servicios a través del teletrabajo y manteniendo reuniones virtuales.

La estrategia de gestión es avalada por el nivel político en este sentido se resolvió aplicar una estrategia de desarrollo humano y en este sentido se comentan procesos de trabajo interesantes. Por ejemplo, se ha creado la Gerencia de Desarrollo Humano otorgándole una impronta totalmente diferente a la anterior donde se está trabajando acerca del desarrollo de competencias; antes había una oficina de personal que liquidaba sueldos y articulaba las búsquedas y los procesos de capacitación conjuntamente con una consultora externa. Se empezaron con prácticas de escuchas que eran necesarias, pero por otro lado suelen 
incomodar. La gerencia de Desarrollo Humano depende de la Gerencia General y está al mismo nivel que el resto de las gerencias.

"Es un proceso de cambio cultural, una vez escuché un dicho que decía a la estrategia la cultura se la come en el desayuno, podes tener la mejor estrategia, pero la cultura la desayuna entonces comenzamos un cambio cultural articulado con la política y la gente lo tiene que comprar lo que se está viendo que hay personas que antes eran invisibles que tienen mucho potencial" (Pablo, 17 de agosto de 2021).

En este contexto la tecnología nos ha favorecido.

"La semana pasada fuimos invitados a una capacitación y había que trabajar por sala, yo trabajé con una asesora de Chipoletti, una sub encargada de oficina de Río Cuarto y una persona de Mendoza, esto antes hubiese sido muy difícil, no todos tienen la posibilidad de viajar, en este sentido aprovechamos las ventajas que nos proporciona la tecnología" (Pablo, 17 de agosto de 2021).

La organización tiene un margen de adaptación a los cambios que se percibe imperioso, dado que el contexto y la realidad del sistema de salud lo exigen y Federada transitó varios años sin "aggionarse". A modo de ejemplo, hasta el año 2018 la mutual se manejaba con un outsorcing informático, no tenía sistema propio y a partir de entonces empezó a armar su equipo de trabajo que a veces sirvió y en otras oportunidades no. En el presente también se está reformulando el sistema tecnológico.

Retomando la consulta en torno a la estrategia de gestión, se destaca que cuando se decidieron implementar los cambios estratégicos participaron de la reflexión colaborados de todas las regiones con diferentes expertise y delegados que tenían liderados y otros que no tenían personal a cargo; así como personas muy noveles y otros con mucha antigüedad en la organización. Se empleó la técnica SMART planteándose objetivos específicos, mensurables, alcanzables, relevantes y temporales.

En la práctica se hicieron instancias de capacitación mostrándose ejemplos, se trabaja sobre los sistemas, no obstante demanda tiempo y esfuerzo porque la gente no tenía hábitos de hablar con sus reportes, identificándose más de cincuenta mandos medios que nunca lideraron gente, registrándose situaciones incómodas para las personas. Años atrás un entrevistado comentó.

"En la mutual hay una camada de empleados más jóvenes que tienen entre veinte y treinta años que arman grupos, salen juntos, son amigos. Hay problemas como en toda empresa, pero se trata de resolverlos hablando, escuchando y después tomando las decisiones correspondientes. En general, es una organización en la cual a la gente le gusta trabajar y la elige para trabajar" (Empleado A; 2014). 
Declaración que evidencia diferencias generacionales y deja entrever ciertas problemáticas no resueltas. Si nos remitimos al historial de la organización se corrobora una estructura verticalista hoy en proceso de cambio. Durante la pandemia, en pos de capacitar a los empleados para tomar decisiones operativas y alentarlos a consultar con la dirección los temas de interés se propuso un "flash informativo". Consiste en proponer un tema y convocatoria a participar por medio de un correo electrónico, luego la gente se suma a través del zoom. Generalmente se realiza los días viernes. En el primer flash intervino el gerente general y en el asunto decía "pregúntame lo que quieras" dejando abierta y alentando la participación.

Hubo quiénes leyeron el mensaje y se empezaron a empoderar construyendo mesas de trabajo, trabajando sobre los emergentes, proponían temas y no tenían temor a decir "podemos hablar de potencial acoso hasta de cosas internas para cambiar cosas en tal región y después lo que ayuda mucho es que realizamos búsquedas internas y descubrimos talentos que están escondidos en puestos que son claves para la organización". La propuesta fue exitosa en el sentido que se fueron presentando situaciones diversas, pero no se logró la participación de la mayoría.

En cuanto al desarrollo de la estructura, se evidenció un crecimiento no previsto, no planeado, por lo tanto, requería realizar un trabajo estratégico de reordenamiento. Hay una propuesta de generar cuatro regiones para administrar, otro cambio importante que está en proceso. Vinculado a las personas y los equipos de trabajo hay mucha gente que quiere ser evaluada, lo cual revela interés y aspiraciones de crecimiento personal.

Se cuenta con equipos de personas efectivas y eficientes siendo los integrantes, especialmente los profesionales más jóvenes, quienes pretenden un plan de carrera y otros están viendo la posibilidad de una sucesión sobre quien gerencia un espacio. Hay muchas personas que se están quedando afuera de la organización y esto no ocurría antes porque no había registro de sanciones ni se evidenciaban los contrastes entre quienes trabajaban mucho y otros que no hacían nada. El vértigo es inevitable, "antes nunca pasaba nada y ahora está pasando" pero se considera positivo. Los cambios se generan acompasados por instancias de diálogo para que comiencen a ser aceptados con naturalidad.

La pandemia fue una oportunidad dado que coadyuvó al aceleramiento para cambiar el modelo de trabajo. Federada estableció un modelo de trabajo mixto. Algunos van a trabajar cien por ciento en la modalidad de teletrabajo porque el BackOffice es bien recibido y no están obligados, es un tema consensuado. Otros continuarán trabajando en un cien por ciento de manera presencial, por ejemplo los servicios esenciales, farmacia, vacunatorio y algún puesto de atención al público o quizás alguna guardia mínima en la organización presencial. 
El proceso de cambio bajo la modalidad virtual se acompañó con un reconocimiento en gasto de teletrabajo, determinándose un monto dentro de los beneficios determinados por no trabajar en forma presencial, por ejemplo, el gasto de almuerzo o el uniforme se prorrateó estipulándose un costo que se traslada como beneficio a los colaboradores y no un ahorro para la empresa.

El Gerente General reconoce que la modalidad de trabajo virtual permitió acortar tiempos y distancias, a modo de ejemplo dijo: "Yo viajaba a Buenos Aires por una reunión de una hora y perdía todo el día, hoy a través de la conectividad cumplimos con los objetivos en menos tiempo y con menos costos". Federada logró una rápida adaptación a los cambios.

Se evidencia un estilo de liderazgo mixto ejercido por un gerente que denota capacidades técnicas, humanas y conceptuales con visión de futuro.

"Ahora vamos por la capacidad de cambio que es más difícil porque estamos apostando a mejorar un montón de cosas que algunas son hozadas para el tipo de entidad, pero necesarias porque si uno mira a un futuro de tres años van a desaparecer muchas de las cosas que se vienen haciendo ahora y hay que empezar ya. El sistema de salud como lo conocemos actualmente, lo que es medicina prepaga, va a terminar siendo virtuoso, de lujo para una población pequeña entonces, ya tenemos que pensar en un producto que sea más abarcativo, no tan caro y que este a la altura de la realidad que estamos viviendo, estamos pensando en productos de financiación de salud con productos de seguros, de personas, seguros de salud de vida de personas" (Pablo, 17 de agosto de 2021).

Con respecto a los motivadores de la gente no han cambiado. Aquellos que se sienten seguro en su puesto o se sienten a la altura de las circunstancias no tienen problemas. Lo que se observa es el compromiso de hacer lo que hay que hacer. Sin embargo, los que se sienten más cuestionados por parte de la organización son los gerentes de mandos medios; esto hace que sean mirados y medidos con la misma vara. Se está procurando amalgamar el equipo gerencial trabajando la confianza mediante un proceso de coaching ejecutivo.

Se interpreta que la asociación mutual está en un proceso de cambio cultural donde los valores sirvieron de marco para dar el puntapié inicial. Por el momento se avanza paso a paso, pero con pie firme. Es fundamental el apoyo político que está convencido, lo pregonan y ayudan con el ejemplo. En este sentido, la contratación del gerente general fue una apuesta fuerte de apertura hacia otra mirada, identificar una necesidad de cambio y contratar por primera vez para un cargo jerárquico a una persona que no ha nacido o pertenecido a la organización. A partir de entonces también se cubrieron los cargos de dos gerencias estratégicas- Desarrollo Humano y Prestaciones- por personas ajenas a la organización. 
En la mixtura de articular lo interno con lo externo se fue generando confianza en los modelos de trabajo al articular la política con la gestión. Actualmente, los tres actores de la política en función están llevando adelante un cambio en el gerenciamiento de la compañía a raíz de reclamos porque se habían paralizado las decisiones estratégicas y a consecuencia de ellos se había perdido reducido la cartera de asociados y consecuentemente los beneficios de la organización. Obvio que no hay recetas ni éxito garantizado lo cierto que esta organización afrontó los vientos de cambios con resultados que hasta el presente son auspiciosos.

\section{REFLEXIONES FINALES}

Los cambios fueron resultantes de decisiones políticas, sin las cuales su implementación hubiera sido imposible. Partiendo de un diagnóstico reflexivo la organización decidió reconstruir su misión y visión coherentes con los objetivos considerados relevantes. En el pasado estaban definidas, pero eran funcionales a los propósitos de la organización.

En principio se procuró acrecentar las fortalezas y estrechar las debilidades para hacer operativos los cambios pretendidos que se dispuso mediante el empleo de tecnologías propias y el reforzamiento sistemático en la gestión del talento humano. El proceso de cambio no fue impulsado por la pandemia si bien se aceleró a consecuencia de la misma. Fue impulsado por el órgano político y aleccionados en conjunto con una gestión apropiada. La redefinición de valores fue ineludible para orientar el rumbo organizacional. Había sido detectado como una debilidad, siete años atrás, cuando se investigó el caso, exponiéndose que numerosas motivaciones e intereses de sus miembros no eran tenidas en cuentas y algunas debilidades en la estrategia de conducción interpretadas como temas pendientes de revisión. Hoy, se entiende que la Mutual situó las acciones necesarias para emprender un camino de aprendizaje y crecimiento organizacional.

La claridad en los compromisos de trabajo, la orientación a resultados específicos, la adaptación a los ambientes personales e institucionales, demuestra que el cambio es posible. Mutual Federada tiene mucho para aportar, hacia adentro de la organización en relación al desarrollo de sus vínculos laborales, y hacia el exterior, fortaleciendo su compromiso con la comunidad por medio de acciones concretas de responsabilidad social.

La figura del Gerente General se lo interpreta como un líder efectivo en relación a la construcción y conducción de equipos de trabajo y como sostiene Kotter es de relevancia porque complementa su labor de gestión, así como señalaba Sarchman que también es clave para dirigir el cambio de los líderes en los mandos medios, cuestión que se aprecia encaminada pero que aún hay mucho por hacer.

Se infiere que el Gerente General-líder posee un "estilo mixto" que combina capacidades de gerenciar y liderar. Demuestra seguridad, confianza y libertad para expresar lo que siente, 
piensa y quiere. La propuesta del "flash informativo" con la consigna "pregúntame lo que quieras" pone de manifiesto una apertura de comunicación y escucha abierta y directa, sin descuidar las funciones y su rol de gerente.

Finalmente, este proceso se interpreta como un auténtico cambio cultural, que se inició con un diagnóstico de la situación existente y llevó a redefinir los valores compartidos y consensuados por todos de los miembros en pos de la construcción conjunta de una floreciente y apropiada cultura organizacional.

\section{REFERENCIAS BIBLIOGRÁFICAS}

Alles, M. (2017). Desarrollo del talento humano basado en competencias. España: Granica S.A.

Cejas, M. (2008). La Formación profesional basada en competencias.Carabobo,

Venezuela: Editorial Consejo de Desarrollo Científico Humanístico de la Facultad de Ciencias Económicas y Sociales.Disponible en:

http://servicio.bc.uc.edu.ve/faces/revista/a12n22/13-22-7.pdf

Chiavenato, I. (2007). Administración de recursos humanos. El capital humano de las organizaciones [8va. Edición] México: McGraw-Hill.

Chiavenato, I. (2009). Gestión del talento humano. México: Mc Graw Hill.

Drucker, P. F. (1999); “Los desafíos de la administración en el siglo XXI”, Buenos Aires, Argentina: Sudamericana.

Goleman, D., Boyatzis, R. y McKee, A. (2009). El líder resonante crea más. [2da. Ed.] Buenos Aires: De Bolsillo.

Kotter, J. P. (2002), “Lo que de verdad hacen los líderes”, en Harvard Business Review Liderazgo, Bilbao, España: Ed. Deusto.

Melamed, A. y Jalife, F. (2020). Tiempos para valientes. Cómo liderar la crisis y la transformación hoy. Ciudad Autónoma de Buenos Aires: Paidós.

Peiró, J. y Soler, A. (2020). "El impulso al teletrabajo durante El Covid-19 y los retos que plantea". Recuperado el 05/6/2021 de: https://www.ivie.es/wpcontent/uploads/2020/05/11.Covid19lvieExpress. El-impulso-al-teletrabajo-duranteel-COVID-19-y-los-retos-que-plantea.pdf

Senge, P. (1992). La quinta disciplina. El arte y la práctica de la organización abierta al aprendizaje. España: Granica.

Sarchman, J. M. (2021) “Habilidades que requieren las empresas en tiempos de pandemia” Revista Profesional y Empresaria (D\&G), Tomo XXII, Junio 2021, Consulta efectuada el 05 de julio de 2021 a través del siguiente sitio: 
http://www.biblioeco.unsa.edu.ar/pmb/opac css/index.php?|v|=notice display\&id=5 $\underline{2538}$

Yin, R. K. (2004). Investigación sobre estudio de casos. Sage Publications: México.

\section{ANEXO: Guión de entrevista}

Se delinearon preguntas abiertas.

Misión, Visión y políticas establecidas que orientan el rumbo estratégico. ¿Cómo determina usted los resultados o metas a alcanzar en su organización? ¿Qué criterios utiliza para ello?

- En relación al contexto acaecido a partir de la pandemia ¿Cómo acompañó la organización este proceso? ¿Qué herramientas brindó? ¿Cómo se comunicó/comunica con sus colaboradores? ¿Ha registrado empoderamiento entre sus colaboradores?

- En relación a la estrategia de gestión -estructura, tecnología y talento humano- prestar atención a capacidad visionaria, flexibilidad, adaptabilidad, manejo de recursos humanos, entre otros.

Equipo de trabajo- rol de liderazgo ¿Cuenta con el equipo necesario para hacer efectivo y eficiente su trabajo? ¿Hay alguien en el trabajo que aliente su mejora? ¿Cree Ud. que sus opiniones son consideradas por su equipo? Detectar estilo de liderazgo a través de interrogantes en relación a: ¿Se trabaja en equipos? ¿Cómo se toman decisiones y se hace operativo bajo nuevas modalidades de trabajo?

Relacionado a la gestión cultural y el cambio ¿Cree Ud. que se mantienen los mismos valores en la empresa? Si/no ¿cuáles y por qué? Indagar ¿Cómo manejan el trabajo entre la vida empresarial y la familiar en los nuevos entornos? Registro de apreciaciones/sensaciones. ¿Qué valores guían el comportamiento de los colaboradores?

- $\quad$ Liderazgo, manejo de equipo, talento humano ¿Ha tenido que entrenar a algún colaborador para ocupar un puesto de más responsabilidad? ¿qué fue lo que usted hizo?, ¿Cómo lo entreno?, ¿cuáles fueron los resultados?

¿Qué hizo/hace Ud. para motivar a sus colaboradores en este contexto actual? ¿Cree Ud. que se han modificado los intereses, expectativas y/o motivaciones del personal? ¿Notó Ud. cambios en actitudes y/o comportamientos de los gerentes de línea media? ¿Cuál/es? Ejemplificar. 
¿Cómo es el grado de compromiso de los colaboradores para con la organización? ¿Tiene confianza respecto a los colaboradores? ¿Se realizan reuniones participativas con el personal? Frecuencia/modalidades. 